\title{
UTILIZACIÓN DE Amaranthus dubius (AMARANTHACEAE) COMO ALTERNATIVA ALIMENTARIA EN CERDO CRIOLLO MESTIZADO
}

\section{USE OF Amaranthus dubius (AMARANTHACEAE) AS ALIMENTARY ALTERNATIVE IN CROSSBREEDED CREOLE PIG}

GARCIA PARRA, LUIS ${ }^{1 *}$ MVZ., RUIZ, SANTIAGO² Biol., MENDOZA, HECTOR ${ }^{3}$ Zootec., PINEDA, HECTOR ${ }^{3}$ Zootec.

${ }^{1}$ Docente Universidad de Sucre, Facultad de Ciencias Agropecuarias, Colombia. ${ }^{2}$ Docente Universidad de Sucre, Dpto. de Biología. ${ }^{3}$ Facultad de Ciencias Agropecuarias, Zootecnia.

*Correspondencia: luisgarcia271@hotmail.com

Recibido: 11-02-2010; Aceptado: 28-07-2010.

\section{Resumen}

Este trabajo se llevó a cabo con el objetivo de evaluar el desempeño productivo de cerdos alimentados con Amaranthus dubius. Se evaluaron dietas a base de materiales alimenticios producidos en la región, con la adición de Amaranthus dubius (bledo de puerco), durante la ceba de cerdos criollos mestizados. Se utilizaron 12 doce animales, con peso medio de $18 \mathrm{Kg}$ distribuidos en tres tratamientos, según diseño estadístico bloques completos al azar. Los animales alimentados con una dieta compuesta de maíz, premezcla mineral y vitamínica, pastoreo y $A$. dubius presentaron mayor ganancia de peso ( $0,278 \mathrm{~g} / \mathrm{día}$ ) que los que recibieron yuca en lugar de maíz (208 g/día). Los animales del grupo control (manejo tradicional) presentaron la menor ganancia de peso (133 g/día). Se destaca el potencial de uso de A. dubius como forraje alternativo en la ceba de cerdos en la región.

Palabras clave: A. dubius, cerdos mestizados, alimentación, ganancia de peso.

\section{Abstract}

This work was carried out with the objective of evaluating the productive acting of pigs fed with Amaranthus dubius. Diets were evaluated with the help of nutritious materials taken place in the region, with the addition of $A$. dubius (Amaranth, spleen), during crossbreed creole pigs liveweigth gain. 12 animals were used, with half weight of $18 \mathrm{Kg}$ distributed in three treatments, according to statistical design with complete random blocks. The animals fed with a diet of corn, vitamins mixture and mineral mixture, shepherding and $A$. dubius presented bigger gain of weight $(0,278 \mathrm{~g} /$ day $)$ that those that received cassava instead of corn (208 g/día). The animals of the control group (with traditional manage) presented the smallest gain of weight $(133 \mathrm{~g} / \mathrm{dí} a)$. Stands out the potential of use of $A$. dubius like alternative forage in it feeds of pigs in the region. 
Key words: $A$. dubius, crossbreeded creole pigs, feeding, weight gain.

\section{Introducción}

Dentro de la explotación pecuaria a nivel de la Costa Atlántica la porcicultura estaría ocupando el segundo lugar en la producción primaria; de acuerdo con las estadísticas de URPA (1999), en el departamento de Sucre solo el 1,5\% de las explotaciones porcinas se maneja de manera tecnificada (razas mejoradas) y el $98.5 \%$ restante de manera tradicional (criollos y criollos mestizados). Entrevistas (no publicadas) con algunos comercializadores de carne de cerdo en el mercado de Sincelejo y de cabeceras municipales aledañas muestran que la demanda de carne porcina está sustentada en las explotaciones de cerdos criollos, mestizos y mejorados.

Amaranthus dubius (bledo de puerco) es una planta de hábitos herbáceos que en condiciones favorables puede alcanzar hasta $1,5 \mathrm{~m}$ de altura, presenta un tallo ramificado con un gran volumen de tejidos parenquimáticos que le permite hacer un importante almacenamiento de agua y de alimento, su vástago tiene un bajo contenido de fibra, sus flores, frutos y hojas son palatables, es una planta que presenta rápido florecimiento y fructificación, característica que le permite tener un elevado potencial reproductivo siendo muy agresiva en los procesos de sucesión ecológica (GRUBBEN y DENTON, 2004). A. dubius posee la característica de desarrollarse y multiplicarse en cualquier tipo de suelo, en bosques, zonas abiertas, a la sombra y al sol (ANTEN y WERGER, 1996). Esta planta tiene una fácil y rápida reproducción debido a la dispersión de sus semillas, las cuales son producidas en grandes cantidades en un corto desarrollo vegetativo. Además, tiene la capacidad de retoñar, reproducirse y sostenerse durante todo el año con bajo suministro de agua, debido a que su sistema radicular es de tipo axonomórfico cuyo eje principal alcanza una longitud que le permite obtener agua de las capas más profundas del suelo vegetal, comparada con otras plantas consideradas como malezas esta particularidad botánica hacen de $A$. dubius una planta susceptible de explotación con bajo manejo controlado (TAPIA, 1997).

Tallos, hojas, flores y frutos de $A$. dubius son favorables para la alimentación de animales domésticos dado a su bajo contenido de fibra y alta palatabilidad, destacándose por su valor proteico (22,7\% en base seca); para el grano entre 13 y $19 \%$ (LEHMANN, 1990) y de 12 a 27 para la planta (STORDAHL et al., 1999; ARELLANO et al., 2004).

Según SLEUGH et al. (2001) el potencial de Amaranthus spp. no ha sido suficientemente estudiado. Algunas cultivariedades de este género son altamente apreciadas como forraje por su rápido crecimiento y su alto contenido proteico (KAUFFMAN, 1992) igualmente se registra su uso para 
alimentación humana (ARELLANO et al., 2004); es una planta ampliamente distribuida tropicalmente y subtropicalmente en África, Asia, Islas del Pacifico, Caribe y América central, al tiempo que se manifiesta que existen pocos reportes de su uso para fines forrajeros o para ensilaje (CERVANTES, 1986). No obstante, la amplia distribución del género que agrupa 70 especies, la mayoría son nativas de América y solo quince especies provienen de otros continentes (ROBERTSON, 1981)

Este trabajo se llevó a cabo con el objetivo de evaluar el desempeño productivo de cerdos criollos mestizados alimentados con Amaranthus dubius.

\section{Materiales y métodos}

El presente trabajo se realizó en el municipio de Morroa, departamento de Sucre, Colombia ( $9^{\circ} 20^{\prime} \mathrm{N}, 75^{\circ} 19^{\prime} \mathrm{O}$ ), situado a $150 \mathrm{msnm}$. Ambientalmente La zona posee un clima Tropical seco con temperaturas entre 24 y $39^{\circ} \mathrm{C}$ y precipitación anual media de 1.200 mm (IGAC, 1969).

Se utilizaron 12 cerdos criollos mestizos (zungo x Duroc $x$ Landrace), con un peso inicial promedio de $18 \mathrm{Kg}$, los animales se distribuyeron al azar en tres tratamientos, según un diseño en bloques completos al azar. El periodo experimental tuvo una duración de 90 días. Los animales se pesaron cada 14 días, para calcular la ganancia de peso.

Se conformaron tres tratamientos (tipos de dieta). El primer tratamiento (To) consistió de una dieta similar a la ofrecida en condiciones de manejo tradicional en la región, compuesta por sobras de comida y desechos agrícolas, más el pastoreo libre. La dieta consistió de lavaza, suero dulce y pastoreo, aclarando que la lavaza estuvo compuesta por conchas de plátano, ñame, yuca y papa cruda, además de sobras procedentes de alimentos de hogares campesinos, que se ofreció a razón de $1 \mathrm{Kg} /$ día, además del suministro de $5 \mathrm{~L}$ diarios de suero dulce por animal. También se dispusieron alimentos procedente de la vegetación circundante, como: pasto colosoana (Bothriochloa pertusa), angletón (Dichantium aristatum), escobilla (Sida cordifolia) y bledo espinoso (Amaranthus spinosus) entre otros, los cuales se ofrecieron a voluntad durante las horas de pastoreo.

La dieta del tratamiento 1 (T1) estuvo compuesta por yuca seca picada adicionada con premezcla comercial de vitaminas y minerales, y A. dubius fresco y a voluntad. A los animales del tratamiento 2 (T2), se les suministro maíz entero más premezcla vitamínica y mineral y $A$. dubius en fresco y a voluntad. Las plantas de $A$. dubius se obtuvieron del medio natural y en pequeños cultivos implantados para este trabajo. 
Se realizó un análisis de varianza para evaluar el efecto de los tratamientos sobre las variables de desempeño (ganancia de peso), usando los datos obtenidos en las diferentes observaciones. El modelo estadístico consideró la media, el efecto del tratamiento y el error experimental. En caso de significancia estadística $(\mathrm{p}<0.05)$ se aplicó la prueba de Duncan para la comparación de las medias de cada tratamiento (ZAR, 1996)

\section{Resultados y discusión}

Los tratamientos afectaron de manera significativa $(p<0.05)$ la ganancia de peso (Tabla 1) de los cerdos, observándose la mayor ganancia en los animales del T2 (maíz + A. dubius + premezcla vitamínica y mineral) como se observa en la Tabla 1.

Tabla 1. Parámetros productivos de cerdos recibiendo dietas con A. dubius durante un periodo 90 días.

\begin{tabular}{|c|c|c|c|c|c|}
\hline \multirow{2}{*}{ Parámetro $(\mathrm{Kg})$} & \multicolumn{3}{|c|}{ Tratamientos } & \multirow[t]{2}{*}{ Valor $\mathbf{p}$} & \multirow[t]{2}{*}{ ESM } \\
\hline & TO & T1 & T2 & & \\
\hline Peso vivo inicial & 18,3 & $18,0 \mathrm{a}$ & 18,5 & - & - \\
\hline Peso vivo final & 30,3 & 36,8 & 43,5 & - & - \\
\hline Ganancia de peso total & $12,0 \mathrm{a}$ & $18,8 \mathrm{~b}$ & $25,0 \mathrm{c}$ & $<0.001-$ & 5.63 \\
\hline Ganancia diaria de peso & $0,133 a$ & $0,208 b$ & $0,278 \mathrm{c}$ & $<0.001-$ & 0.063 \\
\hline
\end{tabular}

${ }^{1}$ Control: dieta tradicional; T1: dieta con yuca, $A$. dubius, premezcla y pastoreo y T2: dieta con maíz, A. dubius, premezcla y pastoreo. Medias con letras diferentes difieren según la prueba de Duncan $(p<0.05) .{ }^{2}$ Valor de probabilidad. ${ }^{3}$ Error estándar de la media.

Estudios previos realizados por SABOGAL et al. (1993) indican que en la ceba de cerdos basada en el suministro de forraje fresco, en particular con ramio más maíz fortificado, se alcanzó una ganancia diaria de peso de $503 \mathrm{~g} / \mathrm{día}$, con cerdos mestizados con baja influencia genética de razas criollas (zungo, cara de palo y casco de mulo).

Para el presente estudio la ganancia diaria de peso para T2 fue de 278 g/día; destacándose que eran animales producto del mestizaje entre razas criollas (zungo, cara de palo y casco de mulo) y algunas razas mejoradas (Landrace y Duroc), presentes en menor proporción. Por otra parte, hay que destacar que SABOGAL et al. (1993) observó un consumo de $3 \mathrm{~kg} / \mathrm{día}$ de ramio y 2,3 $\mathrm{Kg} / \mathrm{día}$ de maíz fortificado, mientras que en los animales del presente estudio el consumo fue de $1,4 \mathrm{Kg}$ de maíz y $1,2 \mathrm{Kg}$ de $A$. dubius, en el T2.

En cuanto a los resultados del T1 no fue factible contrastar, con los resultados de otros estudios que hayan contemplado la utilización de yuca seca más el suministro de forraje fresco. 
En el T2 los animales alcanzaron un consumo de 1,4 Kg de maíz animal/día y $1,2 \mathrm{Kg}$ de $A$. dubius animal/día, siendo el consumo total de 2,6 Kg de alimento para dicho tratamiento. Los animales de esta dieta consumieron $155 \mathrm{~g}$ de proteína animal/día. En el T1 los animales alcanzaron un consumo de $2 \mathrm{Kg}$ de yuca y $1,1 \mathrm{Kg} A$. dubius animal/día, siendo el consumo total de $3,1 \mathrm{Kg}$ de alimento para dicho tratamiento. El consumo en cuanto a proteína fue de $100 \mathrm{~g} /$ animal/día.

Cuando se trabaja con forraje fresco (ramio) en la alimentación de monogástricos, como aves y cerdos, se deben tener en cuenta, principalmente, dos factores: su alto contenido de humedad (74-84\%); y el alto contenido de fibra (17-25\%), los cuales influyen sobre el volumen, impidiendo que el animal pueda consumir los otros nutrientes en las cantidades requeridas. Además, la fibra es altamente indigestible para los cerdos y aves e interfiere con la digestibilidad de los otros nutrientes (SABOGAL et al.; 1993). A. dubius no es ajeno a esta situación dado que contiene un alto porcentaje de humedad (89\%), sin embargo tiene un bajo contenido de fibra. (0,27\%). Tal vez, son estas las razones por las cuales el consumo oscilo entre 1,5 y $2,5 \mathrm{Kg} /$ día de $A$. dubiusk, que puede catalogarse como alto. A pesar del desconocimiento de la digestibilidad de esta especie vegetal es posible que el animal tenga un mayor consumo cuando forrajea. Quedaría por establecer si el consumo voluntario de esta especie durante el pastoreo libre es mayor.

No existen estudios paralelos sobre la utilización de $A$. dubius como alternativa para la ceba de cerdos. Lo que si se conoce es que tiene una alta preferencia y palatabilidad para los cerdos, lo cual se evidencia en el comportamiento alimentario errante en explotaciones extensivas de campesinos y de pequeños productores que hacen uso del recurso natural forrajero. Diversos estudios muestran que Amaranthus spp. Posee cualidades nutricionales superiores que los cereales comunes y que los forrajes de mayor uso (CHEEKE y BRONSON, 1979; ODWONGO y MUGERWA, 1980; YUE et al., 1987; POND y LEHMANN, 1989; LEHMANN, 1990; STORDAHL et al., 1999), sin embargo son necesarios más estudios al respecto.

\section{Referencias}

ANTEN, N.E.R.; WERGER, M.J.A. 1996. Canopy structure and nitrogen distribution in dominant and subordinate plants in a dense stand of Amaranthus dubius L. with a size hierarchy of individuals. Oecologia 105:30-37.

ARELLANO, M.A.L.; ALBARRACIN, G.; ARCE, S.; MUCCIARELLI, S. 2004. Estudio comparativo de hojas de Beta vulgaris con Amaranthus dubius Mart ex Thell. Revista Internacional Botánica Experimental 53:193-197. 
CERVANTES, S.J.M. 1986. Amaranth: El amaranto recurso forrajero Mexicano no aprocechado.Vet. Mex.17:289-296.

CHEEKE, P.R.; BRONSON, J. 1979. Feeding trials with Amaranthus grain, forage and leaf protein concentrations. Pag. 5-11 en: Proc. 2nd Amaranth Conf. Rodale Press, Emmaus, PA.

GRUBBEN, G.J.H.; DENTON, O.A. 2004. Plant Resources of Tropical Africa 2. Vegetables. PROTA Foundation, Wageningen; Backhuys, Leiden; CTA, Wageningen.

IGAC - INSTITUTO GEOGRAFICO AGUSTÍN CODAZZI.1969. Monografía del departamento de Sucre. Oficina de estudios geográficos. Bogotá, Colombia.

KAUFFMAN, C.S. 1992. Realizing the potential of grain amaranth. Food Rev. Intl. 8:5-21.

LEHMANN, J.W. 1990. The potential of grain amaranths in the 1990s and beyond. Pag. 1-7 en: Proc. 4th National Amaranth Symposium: Perspectives on production, processing, and marketing. Minneapolis, MN. 23-25 Aug. 1990. Univ. Minn., St. Paul, MN.

ODWONGO, W.O.; MUGERWA, J.S. 1980. Performance of calves on diets containing amaranthus leaf meal. Anim. Feed. Sci. Tech. 5:193-204.

POND, W.G.; LEHMANN, J.W. 1989. Nutritive value of a vegetable amaranth cultivar for growing lambs. J. Anim. Sci. 67:3036-3039.

ROBERTSON, K.R. 1981. The General of Amarantaceae in the south eastern United States. Journal of The Arnold Arboretum 62 (3):267-314.

SABOGAL, O.R., PORTELA, C.R., POVEDA, M.C.A.; MONCADA, B.A., 1993. Alimentación de cerdos con algunos productos y subproductos agroindustriales. GartnerG., primera edicion, Santafe de Bogotá D.C. (Colombia) Instituto Colombiano Agropecuario ICA. P.p 44 Boletin Divulgativo.

SLEUGH, B.A.; MOORE, J.K.; BRUMMER, E.C.; KNAPP, A.D.; RUSSELL, J.; GIBSON, L. 2001. Forage Nutritive Value of Various Amaranth Species at Different Harvest Dates. Crop Science 41:466-472.

STORDAHL, J.L.; SHEAFFER, C.C.; DICOSTANZO, A. 1999. Variety and maturity affect amaranth forage yield and quality. J. Prod. Agric. 12:249-253.

STORDAHL, J.L.; SHEAFFER, C.C.; DICOSTANZO, A. 1999. Variety and maturity affect amaranth forage yield and quality. J. Prod. Agric. 12:249-253

TAPIA, M. 1997. Cultivos andinos subexplotados y su aporte a la alimentación. 2a Edición. FAO, Oficina Regional para América Latina y el Caribe. Santiago, Chile. 
URPA-. 1999. Secretaría de Desarrollo Económico y Medio Ambiente. Gobernación de Sucre. Sincelejo, Sucre.

YUE, S.X.; SUN, H.L.; CHANG, B.Y.; CHEN, Z.P.; ZUO, J.W. 1987. The nutritional composition of grain amaranth and its potential for utilization. Acta Agron. Sin. 13:151-156.

ZAR, J.H. 1996. Bioestatistical analysis. Third edition. Prentice-Hall, Inc. Englewood Cliff, N.J. 